\title{
A SHORT PROOF OF CASTELNUOVO'S CRITERION OF RATIONALITY
}

\author{
BY \\ WILLIAM E. LANG ${ }^{1}$
}

Dedicated to Professor John Dyer-Bennet on the occasion of his retirement

\begin{abstract}
We give a new proof in positive characteristic of Castelnuovo's criterion of rationality of algebraic surfaces. We use crystalline cohomology and the de Rham-Witt complex as a substitute for the transcendeal methods of Kodaira.
\end{abstract}

Let $X$ be a nonsingular complete algebraic surface over an algebraically closed field $k$. Let $p_{a}(X)=\chi\left(O_{X}\right)-1$ and let $P_{n}(X)=h^{0}\left(X, K_{X}^{\otimes_{n}}\right)$, where $K_{X}$ is the canonical line bundle. These are birational invariants of $X$.

THEOREM 1. $X$ is rational (birationally isomorphic to the projective plane) if and only if $p_{a}(X)=P_{2}(X)=0$.

Over the complex numbers, this is a classical result of Castelnuovo. A modern proof was given by Kodaira [10]. Zariski [16] gave the first proof in characteristic $p>0$; other proofs were given by $\mathbf{M}$. Artin (unpublished) and G. Kurke [11]. In this paper, we give a short proof of the theorem in positive characteristic. Our proof is closely related to Artin's; however, by using crystalline cohomology and the de Rham-Witt complex, we are able to give a better approximation in characteristic $p$ to the elegant Kodaira proof in characteristic zero. Our debt to the work of Nygaard [13] and Artin and Swinnerton-Dyer [3] will be obvious.

After the first version of this paper was written, I learned that S. Mori has also given a new proof of Castelnuovo's criterion, using his theory of extremal rational curves. I believe that this proof complements his, and have indicated briefly in certain places how (according to taste) one may substitute arguments from his proof for those given here.

Proof of TheOREM 1. Serre [14] has given an exposition of Kodaira's proof which shows (in all characteristics) that if $X$ is a nonsingular complete algebraic surface free from exceptional curves of the first kind with $p_{a}(X)=P_{2}(X)=0$, then either $X$ is rational, or $X$ satisfies

(1) $\operatorname{Pic}(X)$ is an infinite cyclic group generated by $K_{X}$,

(2) $|-K|$ consists of irreducible curves of arithmetic genus 1 , and $\operatorname{dim}|-K| \geqslant 1$.

Received by the editors December 14, 1979 and, in revised form, February 19, 1980.

AMS (MOS) subject classifications (1970). Primary 14J10; Secondary 14F30.

Key words and phrases. Algebraic surface, crystalline cohomology, de Rham-Witt complex, rational double point, Brieskorn resolution, elliptic surface.

${ }^{1}$ NSF Postdoctoral Fellow. 
We recall the argument briefly. Using Noether's (Tsen's) theorem, it is enough to find a curve $C$ of arithmetic genus zero such that $C^{2}>0$. The cases $K^{2}<0$, $K^{2}=0$, and $K^{2}>0$ are considered separately, and one finds the curve in the first two cases by geometric arguments using the Riemann-Roch theorem. In the last case, either the curve exists, or (1) and (2) are satisfied. Mori's theory of extremal rational curves simplifies this part of the argument, and simultaneously gives the structure of rational surfaces free from exceptional curves of the first kind.

Kodaira completes the proof when $k=\mathbf{C}$ as follows. Using the exponential sequence $0 \rightarrow \mathbf{Z} \rightarrow O_{X} \rightarrow O_{X}^{*} \rightarrow 0$ and the vanishing of $H^{1}\left(O_{X}\right)$ and $H^{2}\left(O_{X}\right)$, we get an isomorphism $\operatorname{Pic}(X)=H^{1}\left(O_{X}^{*}\right) \cong H^{2}(X, \mathbf{Z})$. Therefore, if (1) holds, $H^{2}(X, \mathbf{Z})$ is infinite cyclic, generated by the first Chern class of $K_{X}$. By Poincaré duality, $K^{2}= \pm 1$. Now we use Noether's formula $\chi\left(O_{X}\right)=\left(K^{2}+e(X)\right) / 12$, where $e(X)$ is the topological Euler characteristic, to conclude that $K^{2}=9$, which is a contradiction.

We find an exact analogue of this proof in characteristic $p$ under the additional assumption that $X$ is supersingular. Recall that a surface is said to be supersingular if $\rho(X)=B_{2}$, where $\rho(X)$ is the rank of the Neron-Severi group, and $B_{2}=$ $\operatorname{dim} H_{\mathrm{et}}^{2}\left(X, \mathbf{Q}_{l}\right)$.

Proposition 1. Let $X$ be a supersingular surface with $p_{a}(X)=P_{2}(X)=0$. Then $X$ does not satisfy (1).

We use the de Rham-Witt complex and some ideas of Nygaard [13]. Recall that the de Rham-Witt complex of a complete nonsingular surface is a complex $W \Omega_{X}^{*}=0 \rightarrow W O_{X} \rightarrow W \Omega_{X}^{1} \rightarrow W \Omega_{X}^{2} \rightarrow 0$ of sheaves of modules over $W(k)(W(k)$ $=$ Witt vectors of $k)$ such that the hypercohomology $\mathbf{H}^{*}\left(W \Omega_{X}^{*}\right)$ is isomorphic to the crystalline cohomology of $X$. From this, we get the slope spectral sequence

$$
E_{1}^{p, q}=H^{q}\left(X, W \Omega_{X}^{p}\right) \Rightarrow H_{\mathrm{crys}}^{p+q}(X / W) .
$$

Furthermore, $H^{q}\left(W O_{X}\right)$ is the Witt vector cohomology defined by Serre [15]. Nygaard [12], [13] has shown that if $X$ is a surface, then the slope spectral sequence degenerates if and only if $H^{2}\left(W O_{X}\right)$ is finitely generated.

Proof of Proposition 1. Since $H^{1}\left(O_{X}\right)=H^{2}\left(O_{X}\right)=0$, the exact sequence

$$
0 \rightarrow W O_{X} \stackrel{V}{\rightarrow} W O_{X} \rightarrow O_{X} \rightarrow 0
$$

shows that $V: H^{2}\left(W O_{X}\right) \rightarrow H^{2}\left(W O_{X}\right)$ is an isomorphism. Since $H^{2}\left(W O_{X}\right)$ is separated in the $V$-topology [15], $H^{2}\left(W O_{X}\right)=0$. We now use the results of [13] to conclude

(a) $H^{0}\left(W \Omega_{X}^{2}\right)=0$, therefore $H_{\text {crys }}^{2}(X / W) \cong H^{1}\left(W \Omega_{X}^{1}\right)$,

(b) $H_{\mathrm{fl}}^{2}\left(X, \mathbf{Z}_{p}(1)\right) \otimes W(k) \cong H^{1}\left(X, W \Omega_{X}^{1}\right)$.

Now we use the hypothesis of supersingularity. Etale Kummer theory [7, §8.2] shows that $N S(X) \otimes \mathbf{Z}_{l} \cong H_{\mathrm{et}}^{2}\left(X, \mathbf{Z}_{l}(1)\right)$, and flat Kummer theory [8, $($ II.5.B)] shows that $N S(X) \otimes \mathrm{Z}_{p} \cong H_{\mathrm{fl}}^{2}\left(X, \mathrm{Z}_{p}(1)\right)$, therefore $N S(X) \otimes W(k) \cong H_{\text {crys }}^{2}(X / W)$. These isomorphisms are compatible with the intersection pairing. Poincare duality (for étale and crystalline cohomology) now implies that the intersection form is 
unimodular. (This idea is due to Illusie, and appears in [13]). Therefore (1) implies that $K_{X}^{2}= \pm 1$, and we get a contradiction from Noether's formula as above.

Now since $H^{1}\left(O_{X}\right)=H^{2}\left(O_{X}\right)=0$, the formal Brauer group $\widehat{\operatorname{Br}}(X)$ [2] exists and is equal to 0. The Artin-Mazur conjecture [2] implies that $X$ is supersingular, so assuming this conjecture, we have a complete proof. Since the conjecture is currently unproved, we give an ad hoc reduction to the supersingular case. (In Mori's proof, the reduction to the supersingular case is made by embedding $X$ into a weighted $\mathbf{P}^{3}$, usng a simplification of the method of Zariski [16], and then lifting to characteristic zero.)

Proposition 2. Then $X$ be a nonsingular complete surface with $p_{a}(X)=P_{2}(X)=$ 0 satisfying (2). Then $X$ is supersingular.

Proof. First, note that $B_{2}-\rho$ is a birational invariant. We may blow up the base points of a generic pencil contained in $|-K|$ to get a map $h: X^{\prime} \rightarrow \mathbf{P}^{1}$ with fibres of arithmetic genus 1 . Then we may blow down exceptional curves of the first kind in the fibres of $h$ to obtain a relatively minimal elliptic (or quasi-elliptic) surface $f: Y \rightarrow \mathbf{P}^{1}$ where $f$ has no multiple fibres and $Y$ is birationally isomorphic to $X$. If $Y$ is quasi-elliptic, it is also supersingular [5, Part III] and we are done. If $Y$ is elliptic, let $g: J \rightarrow \mathbf{P}^{1}$ be the associated Jacobian elliptic surface, i.e., the relatively minimal model of $\operatorname{Pic}^{0}\left(Y / \mathbf{P}^{1}\right)$.

LEMMA $1 . Y$ is supersingular if and only if $J$ is.

Proof. See [5,Part III], or [7].

Let $L=R^{1} f_{*} O_{Y}$, which is a line bundle on $\mathbf{P}^{1}$. From the canonical bundle formula [5, Part II], we find that $\operatorname{deg} L=-\chi\left(O_{Y}\right)=-1$. Since $L$ is canonically isomorphic to the Lie algebra sheaf of $\operatorname{Pic}^{0}\left(Y / \mathbf{P}^{1}\right) \cong \operatorname{Pic}^{0}\left(J / \mathbf{P}^{1}\right), L \cong R^{1} g_{*} O_{J}$ also.

LEMMA 2 (ARTIN AND SWINNERTON-DYER [3]). J lifts formally to characteristic 0.

Proof. We blow down components of fibres of $J$ not meeting the zero-section $S$ to obtain a surface $J_{0}$ with only rational double points such that all fibres of $g^{\prime}$ : $J^{\prime} \rightarrow \mathbf{P}^{1}$ are irredicible. Let $W=g_{*}^{\prime} O_{J^{\prime}}(3 S)$. Then it is well known that $J^{\prime}$ is defined as a subscheme of $\mathbf{P}(W)$ be a Weierstrass equation, which locally looks like

$$
a_{0}^{\prime} y^{2} z+a_{1} x y z+a_{3} y z^{2}=a_{0} x^{3}+a_{2} x^{2} z+a_{4} x z^{2}+a_{6} z^{3} \text {. }
$$

The patching data defines the $a_{i}$ as a section of $\operatorname{Symm}^{3}(W) \otimes L^{-6}$. W has a canonical filtration [6] such that the associated graded sheaf is isomorphic to $O \oplus L^{2} \oplus L^{3}$. Since in our case $L \cong O_{\mathbf{P}^{1}}(-1), W \cong O \oplus L^{2} \oplus L^{3}$, and each $a_{i}$ is a section of $L^{-i}$, with $a_{0}^{\prime}=a_{0}=1$. To lift $J^{\prime}$ to a surface over a complete discrete valuation ring of characteristic 0 with residue field $k$, we need only lift the $a_{i}$. By imposing a nondegeneracy condition on the lifted $a_{i}$, we can choose the lifting so that the lifted surface has only finitely many rational double points also.

Now Artin's theory of Brieskorn resolutions [1] (simultaneous resolution of rational double points) shows that we can find a Henselian discrete valuation ring $B$, finite over $A$, and an algebraic space $\mathfrak{\Im}$ proper and smooth over $B$ such that the special fibre of $\mathfrak{J}$ is $J$. If we let $J_{n}=\mathfrak{\Im} \times_{B} B / m^{n}$, then each $J_{n}$ is a scheme 
(infinitesimal deformations of schemes are schemes) and we get a formal lifting over $\operatorname{Spf} \hat{B}$.

We now finish the proof easily. Using the Leray spectral sequence, we find that $H^{2}\left(J, O_{J}\right) \cong H^{1}\left(\mathbf{P}^{1}, R^{1} g_{*} O_{J}\right) \cong H^{1}\left(\mathbf{P}^{1}, O_{\mathbf{P}^{1}}(-1)\right)=0$. Therefore, we may lift a very ample line bundle on $J$ to $\mathfrak{\Im}$. Therefore $\mathfrak{\Im}$ is projective over $\hat{B}$. Therefore by the Grothendieck existence theorem, $\mathfrak{\Im}$ is algebraizable, and we may find a scheme $\mathfrak{S}^{*}$ proper and smooih over $\hat{B}$ such that the special fibre is $J$. By upper semicontinuity of cohomology, the geometric genus of the geometric general fibre $\mathfrak{\Im}_{g}^{*}$ is 0 , therefore $\mathfrak{\Im}_{g}^{*}$ is supersingular. But $B_{2}\left(\mathfrak{\Im}_{g}^{*}\right)=B_{2}(J)$, and $\rho$ is upper semicontinuous, therefore $J$ is supersingular. This completes the proof of Proposition 2, and Proposition 1 completes the proof of the theorem.

\section{REFERENCES}

1. M. Artin, Algebraic construction of Brieskorn's resolutions, J. Algebra 29 (1974), 330-348.

2. M. Artin and B. Mazur, Formal groups arising from algebraic varieties, Ann. Sci. École Norm. Sup. (4) 10 (1977), 87-132.

3. M. Artin and H. P. F. Swinnerton-Dyer, The Shafarevich-Tate conjecture for pencils of elliptic curves on $K 3$ surfaces, Invent. Math. 20 (1973), 249-266.

4. E. Bombieri and D. Husemoller, Classification and embeddings of surfaces, Proc. Sympos. Pure Math., vol. 29, Amer. Math. Soc., Providence, R. I., 1975, pp. 329-420.

5. E. Bombieri and D. Mumford, Enriques' classification of surfaces in characteristic p. Part II; Complex analysis and algebraic geometry, Cambridge Univ. Press, 1977, pp. 23-42; Part III; Invent. Math. 35 (1976), 197-232.

6. P. Deligne, Courbes elliptiques, formulaire, d'apres J. Tate, Lecture Notes in Math., vol. 476, Springer-Verlag, Berlin and New York, 1975, pp. 53-73.

7. A. Grothendieck, Le groupe de Brauer. III: Dix exposés sur la cohomologie des schémas, North-Holland, Amsterdam, 1968, pp. 88-188.

8. L. Illusie, Complexe de de Rham-Witt et cohomologie cristalline, Ann. Sci. École Norm. Sup. (4) 12 (1979), 501-661.

9. D. Knutson, Algebraic spaces, Lecture Notes in Math., vol. 203, Springer-Verlag, Berlin and New York, 1971.

10. K. Kodaira, On the structure of complex analytic surfaces. IV, Amer. J. Math. 90 (1968), $1048-1066$.

11. G. Kurke, On Castelnuovo's criterion for rational surfaces, Proc. Internat. Sympos. Algebraic Geometry, Kyoto, 1977, pp. 557-563.

12. N. Nygaard, Closedness of regular 1-forms on algebraic surfaces, Ann. Sci. Ecole Norm Sup. (4) 12 (1979), 33-45.

13. A p-adic proof of the non-existence of vector fields on $K 3$ surfaces, Ann. of Math. 110 (1979), 515-528.

14. J.-P. Serre, Critère de rationalité pour les surfaces algébriques (d'après un cours de K. Kodaira), Séminaire Bourbaki, vol. 9, 1956/57, Exposé 146, Secrétariat Math., Paris, 1959.

15. , Sur la topologie des variétés algébriques en caractéristique p, Sympos. Internac. Topología Algebraica, Univ. Nac. Antónoma de Mexico and UNESCO, Mexico City, 1958, pp. 24-53.

16. O. Zariski, On Castelnuovo's criterion of rationality $p_{a}=P_{2}=0$ of an algebraic surface, Illinois $\mathrm{J}$. Math. 2 (1958), 303-315.

Department of Mathematics, Massachusetts Institute of Technology, Cambridge, MasSACHUSETTS 02139 